\title{
Fragile Self and Malevolent Others: Biased Attribution Styles in Individuals at Ultra-High Risk for Psychosis
}

\author{
Hye Yoon Park ${ }^{1,2}$, Minji Bang ${ }^{3}$, Kyung Ran Kim, ${ }^{1,2}$, Eun Lee ${ }^{1,2}$, and Suk Kyoon $A n^{1,2,4} \bowtie$ \\ ${ }^{1}$ Department of Psychiatry, Yonsei University College of Medicine, Severance Hospital, Seoul, Republic of Korea \\ ${ }^{2}$ Section of Self, Affect and Neuroscience, Institute of Behavioral Science in Medicine, Yonsei University College of Medicine, \\ Seoul, Republic of Korea \\ ${ }^{3}$ Department of Psychiatry, CHA Bundang Medical Center, CHA University, Seongnam, Republic of Korea \\ ${ }^{4}$ Graduate Program in Cognitive Science, Yonsei University, Seoul, Republic of Korea
}

\begin{abstract}
Objective Biased attribution styles of assigning hostile intention to innocent others and placing the blame were found in schizophrenia. Attribution styles in individuals at ultra-high risk (UHR) for psychosis, however, have been less studied especially for its association with various psychological factors. We investigated whether UHR individuals show increased hostility perception and blaming bias and explored the associations of these biased styles of attribution with the factor structure of multifaceted self-related psychological variables and neurocognitive performances.

Methods Fifty-four UHR individuals and 80 healthy controls were assessed by evaluating resilience, self-perception, self-esteem, and aberrant subjective experiences of schizotypy (physical anhedonia, social anhedonia, magical ideation, and perceptual aberration), basic symptoms, and carrying out a comprehensive neurocognitive test battery. Attribution styles were assessed using the Ambiguous Intentions Hostility Questionnaire.

Results UHR individuals, compared with normal controls, showed increased hostility perception and blaming bias. Factor analysis of self-related psychological variables and neurocognitive performances in the entire subject population showed a three-factor solution, which was designated as reflective self, pre-reflective self, and neurocognition. Multiple regression analysis in UHR individuals revealed that hostility perception bias was associated with reflective self and composite blame bias was associated with reflective and pre-reflective self.

Conclusion This study supports the emergence of attribution biases in the putative 'prodromal' phase of schizophrenia. The associations of biased attribution styles with multifaceted self-related psychological constructs suggest that psychosocial interventions for biased attribution styles in UHR individuals should focus not only on reflective self but also pre-reflective self-related psychological constructs.
\end{abstract}

Psychiatry Investig 2018;15(8):796-804

Key Words Attribution style, Ultra-high risk for psychosis, Reflective self, Pre-reflective self, Neurocognition.

\section{INTRODUCTION}

Human beings constantly communicate with the world and in the process try to understand the meanings of and reasons for the events occurring in the surroundings and attempt to provide explanations. While inferring the meaning of the events, which may not be expressly stated, the attribu-

\footnotetext{
Received: February 1, 2018 Revised: April 3, 2018

Accepted: May 8, 2018

$\square$ Correspondence: Suk Kyoon An, MD, PhD

Department of Psychiatry, Yonsei University College of Medicine, 50-1 Yonsei-ro, Seodaemun-gu, Seoul 03722, Republic of Korea

Tel: +82-2-2228-1585, Fax: +82-2-313-0891, E-mail: ansk@yuhs.ac

(c) This is an Open Access article distributed under the terms of the Creative Commons Attribution Non-Commercial License (https://creativecommons.org/licenses/bync/4.0) which permits unrestricted non-commercial use, distribution, and reproduction in any medium, provided the original work is properly cited.
}

tion style determines the individual's manner of assigning causality for the outcomes. ${ }^{1}$ Normal individuals generally tend to display a self-serving bias, which is the attribution of positive events to themselves (internal attribution) and negative events to external factors (external attribution). ${ }^{2}$ In paranoid patients, a greater tendency to blame others for negative events is exhibited. ${ }^{3}$ In schizophrenia, a biased attribution style-which regards others as malevolent-was presented in not only multiple-episode patients ${ }^{4}$ but also first-episode patients and prodromal phase participants. ${ }^{5}$

Attribution may possibly be related to a number of factors since individuals' action and perception towards the world have been attempted to be understood by a conjoint of multiple psychological components. ${ }^{6}$ Neurocognitive functioning is responsible not only for perception and attention but 
also for higher-order inferential processes. ${ }^{7}$ Episodic memory and experiential knowledge may be used to compare past experiences to a current situation when making judgments of events. ${ }^{8}$ Executive functions may help to find and choose adequate explanations for the causes of happenings. ${ }^{9}$ In individuals with delusions, the lower availability of alternative explanations are related to the higher delusional conviction. ${ }^{10}$ In schizophrenia patients, neurocognitive deficits including memory functions, psychomotor speed, and executive function were found to be the key contributory factors for misattribution. ${ }^{11,12}$ Apart from neurocognition, recently, aspects of self have been reported to serve as accounting factors for attribution. For example, Bentall pointed out the importance of selfdiscrepancies (the gap between actual self and ideal self) for determining attributions. ${ }^{1}$ Research on self-related psychological variables including self-esteem were extensively reported to be associated with abnormal reasoning. Low self-esteem was suggested to be defended by the role of the selfserving bias. ${ }^{13} \mathrm{~A}$ study on self-esteem and self-efficacy in relation to a composite index of attribution of Attributional Style Questionnaire $\left(\mathrm{ASQ}^{14}\right)$, which was increased with internal, global, and stable explanations resulted in positive correlations on positive events and negative correlations on negative events in healthy subjects. ${ }^{15}$ In participants suffering from persecutory delusions ${ }^{16}$ and in schizophrenia patients, ${ }^{17}$ negative selfconcept was associated with paranoia, which is an exaggerated example of misattribution.

In the clinical picture of schizophrenia, research on aspects of self has been focused to understand various anomalies of subjective experience. A disturbance of subjective experience in schizophrenia reflects a disrupted basic sense of self, a distorted sense of being a subject of experiences. ${ }^{18}$ Studies of anomalous self experience have led to research on frameworks of schizotypy ${ }^{19}$ and the basic symptoms, ${ }^{20}$ both of which were suggested to result from deficits in information processing. ${ }^{18}$ Based on above association with self, schizotypy and basic symptoms could be likely considered as one of the important facets of self-related psychological variables for psychosis. The core components of schizotypy (e.g., perceptual aberration, magical ideation, and anhedonia) were introduced to understand psychosis-proneness and the pathological process in schizophrenia spectrum disorders. ${ }^{21}$ The degree of schizotypy was correlated with the tendency for judging the action of others to be intentional in normal subjects. ${ }^{22} \mathrm{~A}$ high level of social anhedonia revealed deficits in inferring the beliefs of others in schizophrenia patients. ${ }^{23} \mathrm{~A}$ high level of the perceptual aberration was found to be negatively correlated with the recognition ability to the emotional state of others in firstepisode schizophrenia patients and putative prodromal individuals. ${ }^{24}$ Basic symptoms, another concept to explain early self-experienced changes in psychotic patients, are defined as subclinical disturbances in mental processes. ${ }^{20}$ Basic symptoms contain wide ranges of dysfunctions in domains such as thought, perception, action and memory, and these disturbances could only be identified by the affected person. ${ }^{25} \mathrm{Ba}$ sic symptoms have been reported to be a main factor for the formation of paranoid ideation in schizophrenia. ${ }^{9,26}$ Because the generation of paranoid idea is closely related to attribution, 1 basic symptoms may possibly be considered as one of the possible factors for attribution style.

The phenomenological tradition approaches the self by focusing on two distinct levels: Pre-reflective self and reflective self. ${ }^{27}$ The self needs to be confident about the first-person givenness of experiences to be the subject of the dialogue with the world. This pre-reflective aspect of the self is called the basic self, minimal self, or ipseity. ${ }^{28}$ The pre-reflective self enables us to feel an immediate sense of self-presence and selfas-subject. ${ }^{27}$ Besides the pre-reflective self, the explicit awareness of the self may affect the individual to realize his or her continuing identity in the world. ${ }^{27,28}$ This reflective level of the self is regarded as the narrative self. ${ }^{29}$ The self-related psychological variables, which might affect self to be psychologically fragile, such as self-esteem, self-efficacy, self-perception, schizotypy, and basic symptoms may also be classified into two aspects of self. As the basic form of self-experience is manifested implicitly and pre-reflexively, ${ }^{30}$ the variable of basic symptoms is assumed to have a characteristic of pre-reflective self. A strong relationship between schizotypy and anomalous selfexperience is also suggested. ${ }^{31}$ The fact that questionnaires of schizotypy or self-experience showed several common features (perceptual disturbances, thought disorders, magical ideation, etc.), ${ }^{32}$ schizotypy is also closely related to the pre-reflective level of self. Since the reflective self covers all aspects of an individual's personality, ${ }^{27}$ resilience, self-perception, and selfesteem are included in this level of self. Overall, the multifaceted self-related psychological and neurocognitive performance variables, which are related to infer the causality of experiences may be comprehensively understood by the perspective of three dimensions; two levels of self and neurocognition.

To provide better insight of the characteristics of a biased attribution style in schizophrenia, prodromal phase individuals need to be evaluated as attributional biases have already emerged in these before they are in an overtly psychotic state $^{5}$ as well as undesirable effects including long-term uses of antipsychotic medications and the secondary influence of chronic illness can be ruled out. ${ }^{33}$ Ultra-high risk (UHR) individuals for psychosis, those with attenuated psychotic symptoms along with functional decline, ${ }^{34}$ are a major research priority for schizophrenia in the pre-onset phase. Exploring the self-world interaction with the viewpoint of the three dimen- 
sions, two levels of self and neurocognition, in UHR individuals may contribute to the search for the verities of schizophrenia itself since abnormal self-experience is one of the central features in the prodromal phase of schizophrenia. ${ }^{35}$

A few studies have examined the biased attribution style in high-risk subjects. ${ }^{5,36,37}$ The studies have found a preference for perceiving hostility and blaming others, ${ }^{5}$ exhibiting externalized locus of control ${ }^{37}$ in UHR individuals, although another study did not confirm the significant attribution bias in clinical high-risk patients. ${ }^{36}$ The biased attribution style, however, has barely been investigated for its relation to various psychological factors in UHR individuals. The present study aims at: 1) demonstrating the biased attribution styles in UHR individuals, 2) identifying the factor structure of the multifaceted self-related psychological variables and neurocognitive performance variables, and 3) investigating whether the biased attribution styles are associated with the aboveidentified factors. We hypothesized firstly that the UHR individuals will exhibit biased attribution styles (replication study 5). Secondly, the multifaceted self-related psychological and neurocognitive performance variables would be categorized into three factors for the entire subject population [healthy controls (HCs) and UHR individuals], and these factors may be represented and verified as possessing the respective characteristics of reflective self, pre-reflective self, and neurocognition. Finally, we hypothesized that there will be associations with biased attribution styles and the three factors in individuals at UHR.

\section{METHODS}

\section{Participants}

Fifty-four UHR individuals and $80 \mathrm{HCs}$ participated in the present study. The present study was a part of the Green Program for Recognition and Prevention of Early Psychosis project at the Severance Hospital of the Yonsei University Health System and details of inclusion and exclusion criteria are described elsewhere. ${ }^{5}$ All participants were recruited between October 2007 and September 2016 and assessed for psychiatric disorders using the Structured Clinical Interview for DSM-IV (SCID-I). ${ }^{38,39}$ UHR individuals were diagnosed according to the Criteria of the Prodromal Syndromes from the Structured Interview for Prodromal Syndromes (SIPS). ${ }^{40}$ Individuals at UHR met one or more of the following criteria: 1) attenuated psychotic symptoms (APS), defined by development or worsening of attenuated positive psychotic symptoms within the past year, 2) brief intermittent psychotic symptoms (BIPS), characterized by experiencing frank psychotic symptoms which are spontaneously remitted within one week, or 3) genetic risk and deterioration syndrome
(GRDS), defined as having a genetic risk as well as a recent functional decline.

The present study was carried out in accordance with the Declaration of Helsinki (2008). The Institutional Review Boards at the Severance Hospital and Severance Mental Health Hospital approved this study (4-2014-0744, 4-2016-0570). All participants and the parents of subjects who were under 18 years of age gave written informed consent. Table 1 shows the demographic and clinical profiles of the participants.

\section{Measures}

\section{The multifaceted self-related psychological variables}

The multifaceted self-related psychological variables were assessed in the areas of resilience, self-perception, self-esteem, features of schizotypy, and basic symptoms which may influence the self to be fragile. Details on the assessments of above variables variables-the Connor-Davidson Resilience Scale, ${ }^{41}$ Self-Perception Scale, ${ }^{42}$ Rosenberg's Self-Esteem Scale, ${ }^{43}$ Chapman's true-false self-report questionnaires for physical anhedonia, ${ }^{44}$ social anhedonia, ${ }^{45}$ magical ideation, ${ }^{46}$ and perceptual aberration, ${ }^{47}$ and the Frankfurt Complaint Questionnaire ${ }^{48}$-are provided in the Supplementary Material (in the online-only Data Supplement).

\section{Neurocognitive performance variables}

A comprehensive neurocognitive test battery that is described in our previous publication ${ }^{49}$ was used to assess neurocognitive function. The battery consisted of the California Verbal Learning Test, Rey Complex Figure Test, Controlled Oral Word Association Test, Figure Fluency Test, Trail Making Test Part A and B, 3-7 Continuous Performance Test, Verbal and Spatial 2-back Test, Wisconsin Card Sorting Test, and Stroop Test. The test scores were converted to $\mathrm{z}$ scores based on the performance of $\mathrm{HC}$ subjects to compare the different neurocognitive test results with standard metrics. Five dimensions were determined on the confirmation of the factor structure: $:^{50}$ verbal memory, spatial memory, processing speed, attention/ working memory, and executive function. The internal consistencies of verbal memory (0.89), spatial memory (0.97), processing speed (0.60), attention/working memory (0.75), and executive function (0.54) were comparable in the present study. The mean of the classified test scores were used as the summary scores of each domain.

\section{Attribution style}

The attribution style was assessed using the Ambiguous Intentions Hostility Questionnaire (AIHQ). ${ }^{51,52}$ The AIHQ is a self-report scale, which is composed of hypothetical negative situations with varied intentionality: five accidental, 5 ambig- 
uous, and 5 intentional scenarios. The AIHQ yields scores of the hostility perception bias, the composite blame bias, and the aggressive response bias. Subjects were asked to read each vignette, to imagine them in the situation, and to write down the reason for the behavior of the other person (or persons) towards them. The hostility index, rated by the rater according to the written response, ranged from 1 ("not at all hostile") to 5 ("very hostile"). Intentionality [range 1-6; "Did the other person (or persons) act that way on purpose?"], blame [range 1-5; "How much would you blame the other person (or persons)?"], and anger (range 1-5; "How angry would you be?") were rated by the participant, and a mean score of these three items were calculated to provide the composite blame bias score (range 1-5.3). The aggressive response bias is a rater-driven score of the participant's response to the situation including both verbal and physical aggression, which ranged from 1 ("not at all aggressive") to 5 ("very aggressive").

As the ambiguous items need to be rated in the context of the accidental and intentional scenarios, subjects were administered all 15 items. In this study, only three bias scores for ambiguous situations (items 3, 5, 8, 10, and 13) were used according to the analysis strategy of previous studies. ${ }^{4,52}$ Cronbach's $\alpha$ for the composite blame bias in ambiguous situations was 0.74 in the current study.

\section{Other clinical measures}

If the multifaceted self-related psychological and neurocognitive performance variables are indeed three factor models characterized by reflective self factor, pre-reflective self factor, and neurocognition factor, this would suggest that the factors may show unique correlations with other variables. To verify these correlations, self-directedness (SD), self-transcendence (ST), and intelligence quotient (IQ) are also measured. Details on the assessments of SD, ST, and IQ-SD and ST dimensions in the character section of the Temperament and Character Inventory, ${ }^{53-56}$ and the Korean-Wechsler Adult Intelligence Scale ${ }^{57}$-are provided in the Supplementary Material (in the online-only Data Supplement).

The presence of psychopathology and the severity of symptoms were evaluated using the positive and negative syndrome scale (PANSS). ${ }^{58}$

\section{Statistical analysis}

Independent $\mathrm{t}$-tests and $\chi^{2}$ tests were used to examine group differences in the demographic and clinical characteristics. The biased attribution styles were compared using the independent $\mathrm{t}$-test between two groups.

To study the factor structure of psychological constructs, exploratory factor analysis was carried out. We completed principal component analyses with the multifaceted self-related psychological and neurocognitive performance variables for the entire subject population ( 54 UHRs, $80 \mathrm{HCs}$ ). The number of factors was considered on the basis of the scree plot, and factors with eigenvalues greater than or equal to 1.0 were determined to be useful and submitted to orthogonal rotation. Items with factor loadings greater than or equal to 0.4 were considered to be significant. An item with a loading of $\geq 0.4$ for more than one factor was assigned to the factor with higher loading. Factor scores were then derived for use in correlation and multiple regression analyses.

Correlations among self-directedness, self-transcendence, IQ and the factors were calculated using Pearson's correlation analysis. The preliminary analysis of the relationships between AIHQ and the factors were also assessed using Pearson's correlation. Post-hoc comparisons with Bonferroni correction were done for both multiple comparisons ( $\mathrm{p}<0.05 / 9$ i.e., $<0.006$ ).

All variables significantly related to the subdomain scores of AIHQ were analyzed subsequently using a multiple linear regression analysis to evaluate their independent contributions to attribution style. The regression model utilized an enter method. The criterion for significance was set at $\mathrm{p}<0.05$.

\section{RESULTS}

\section{Comparison of demographic and clinical characteristics between UHR and HC groups}

There were no significant differences in sex, age, or years of education as shown in Table 1. The UHR and HC groups were found to show significant differences in all 13 multifaceted self-related psychological and neurocognitive variables except the executive function test scores (Table 1).

\section{Comparison of AIHQ scores in ambiguous items between UHR individuals and HCs}

When the UHR and HC groups were compared with respect to attribution style, the UHR individuals showed significantly higher AIHQ perceived hostility and composite blame bias, and significantly lower aggressive response bias scores of the ambiguous situations as shown in Table 2.

\section{Factor structure of the multifaceted self-related psychological and neurocognitive performance variables}

Principal component analysis to reduce the number of predictor variables resulted in three factors with eigenvalues of $4.99,1.72$, and 1.37 , which together accounted for $62 \%$ of the variance $(\mathrm{KMO}=0.83)$. The loadings of the multifaceted selfrelated psychological and neurocognitive variables on the three factors are shown in Table 3. High loadings on Factor 1 were from great resilience, positive self-perception, high self-esteem, 
and less anhedonia. Factor 2 received high loadings from elevated magical ideation and perceptual aberration scores, and high levels of basic symptoms. Factor 3 obtained significant loadings from the ability to perform all the neurocognitive tests. Based on the distribution of variables, Factor 1 was named as "reflective self," Factor 2 as "pre-reflective self," and

Table 1. Demographic and clinical characteristics of the study groups

\begin{tabular}{|c|c|c|c|c|}
\hline & \multirow{2}{*}{ UHR $(\mathrm{N}=54)$} & \multirow{2}{*}{$\mathrm{HC}(\mathrm{N}=80)$} & \multicolumn{2}{|c|}{ Statistical analysis } \\
\hline & & & Value & $\mathrm{p}$ \\
\hline Sex: male/female & $31 / 23$ & $38 / 42$ & $\chi^{2}=1.28$ & 0.293 \\
\hline Age (years) & $20.5(3.6)$ & $20.7(3.4)$ & $\mathrm{t}=0.21$ & 0.838 \\
\hline Educational level (years) & $13.2(1.9)$ & $13.2(2.0)$ & $\mathrm{t}=0.13$ & 0.895 \\
\hline \multicolumn{5}{|l|}{ SIPS-defined prodromal status (N) } \\
\hline APS & 38 & & & \\
\hline BIPS & 1 & & & \\
\hline APS+BIPS & 4 & & & \\
\hline APS+GRDS & 13 & & & \\
\hline \multicolumn{5}{|l|}{ Self-related psychosocial variables } \\
\hline Resilience of CD-RISC & $40.7(13.6)$ & $62.2(13.3)$ & $t=9.07$ & $<0.001$ \\
\hline Self-perception of Self-Perception Scale & $51.8(14.9)$ & $71.8(15.0)$ & $\mathrm{t}=7.58$ & $<0.001$ \\
\hline Self-esteem of RSE & $22.6(5.2)$ & $29.9(4.7)$ & $\mathrm{t}=8.43$ & $<0.001$ \\
\hline Social anhedonia of Chapman's Scale & $22.9(8.5)$ & $9.6(5.4)$ & $\mathrm{t}=-10.26$ & $<0.001$ \\
\hline Physical anhedonia of Chapman's Scale & $25.1(11.7)$ & $13.6(7.4)$ & $t=-6.39$ & $<0.001$ \\
\hline Magical ideation of Chapman's Scale & $10.3(6.2)$ & $6.5(4.4)$ & $\mathrm{t}=-3.92$ & $<0.001$ \\
\hline Perceptual aberration of Chapman's Scale & $7.9(6.6)$ & $4.1(4.8)$ & $t=-3.63$ & $<0.001$ \\
\hline Basic symptoms of FCQ & $36.1(21.8)$ & $10.0(13.7)$ & $\mathrm{t}=-7.81$ & $<0.001$ \\
\hline \multicolumn{5}{|l|}{ Neurocognitive performance* } \\
\hline Verbal memory & $-0.6(1.2)$ & $0.0(0.9)$ & $\mathrm{t}=3.33$ & 0.001 \\
\hline Spatial memory & $-0.3(0.9)$ & $0.0(0.9)$ & $\mathrm{t}=2.05$ & 0.043 \\
\hline Processing speed & $-0.4(0.7)$ & $0.1(0.7)$ & $\mathrm{t}=3.81$ & $<0.001$ \\
\hline Attention/working memory & $-0.4(0.9)$ & $0.0(0.7)$ & $t=2.65$ & 0.009 \\
\hline Executive function & $-0.2(0.8)$ & $0.0(0.7)$ & $\mathrm{t}=1.58$ & 0.116 \\
\hline \multicolumn{5}{|l|}{ PANSS $^{\dagger}$} \\
\hline Positive symptoms of PANSS & $14.0(3.8)$ & & & \\
\hline Negative symptoms of PANSS & $18.2(6.0)$ & & & \\
\hline General psychopathology of PANSS & $34.9(6.2)$ & & & \\
\hline \multicolumn{5}{|l|}{ Antipsychotic medications } \\
\hline Naive/medicated & $38 / 16$ & & & \\
\hline Chlorpromazine equivalent (mg/d) & $103.3(65.3)$ & & & \\
\hline
\end{tabular}

${ }^{*}$ neurocognitive performance data are analyzed with z score, ${ }^{+}$PANSS data available for 53 UHR participants. SIPS: Structured Interview for Prodromal Syndromes, APS: attenuated psychotic symptoms, BIPS: brief intermittent psychotic symptoms, GRDS: genetic risk and deterioration syndrome, CD-RISC: Connor-Davidson Resilience Scale, RSE: Rosenberg's Self-Esteem Scale, FCQ: the Frankfurt Complaint Questionnaire, PANSS: positive and negative syndrome scale, UHR: ultra-high risk, HC: healthy controls

Table 2. Comparison of AIHQ scores in ambiguous items between UHR individuals and HCs

\begin{tabular}{lccc}
\hline & UHR $(\mathrm{N}=52)$ & HC $(\mathrm{N}=80)$ & $\mathrm{p}$ \\
\hline Hostility perception bias & $2.2(0.7)$ & $1.6(0.5)$ & $<0.001$ \\
Composite blame bias & $3.3(1.1)$ & $2.4(0.7)$ & $<0.001$ \\
Aggressive response bias & $1.6(0.6)$ & $1.9(0.4)$ & 0.006 \\
\hline
\end{tabular}

AIHQ: Ambiguous Intentions Hostility Questionnaire, UHR: ultra-high risk, HC: healthy controls 
Table 3. Loadings on factors derived by principal component analysis with orthogonal rotation

\begin{tabular}{lccc}
\hline & Factor 1: reflective self & Factor 2: pre-reflective self & Factor 3: neurocognition \\
\hline Resilience & $0.86^{*}$ & -0.10 & 0.18 \\
Self-perception & $0.84^{*}$ & -0.12 & 0.09 \\
Self-esteem & $0.84^{*}$ & -0.19 & 0.08 \\
Social anhedonia & $-0.79^{*}$ & 0.28 & -0.11 \\
Physical anhedonia & $-0.78^{*}$ & 0.18 & -0.08 \\
Magical ideation & -0.18 & $0.87^{*}$ & -0.07 \\
Perceptual aberration & -0.19 & $0.84^{*}$ & -0.09 \\
Basic symptoms & $-0.52^{*}$ & $0.75^{*}$ & -0.08 \\
Verbal memory & 0.03 & -0.10 & $0.71^{*}$ \\
Spatial memory & 0.04 & 0.28 & $0.64^{*}$ \\
Processing speed & 0.37 & -0.04 & $0.58^{*}$ \\
Attention/working memory & 0.15 & -0.08 & $0.56^{*}$
\end{tabular}

For this table, all loadings are represented such that positive loadings indicate better scores on the item. *loadings $>0.40$

Table 4. Correlations of the three factors with self-directedness, self-transcendence, and IQ

\begin{tabular}{lccc}
\hline & Reflective self factor & Pre-reflective self factor & Neurocognition factor \\
\hline Self-directedness & $0.61(<0.001)$ & $-0.34(<0.001)$ & $0.13(0.147)$ \\
Self-transcendence & $0.17(0.050)$ & $0.50(<0.001)$ & $-0.02(0.841)$ \\
IQ & $0.12(0.176)$ & $0.00(0.970)$ & $0.54(<0.001)$ \\
\hline
\end{tabular}

Due to the number of correlations, a corrected probability level was set a priori at 0.006 (i.e., $0.05 / 9$ ), and correlations falling above that level were considered to be nonsignificant. IQ: intelligence quotient

Table 5. Multiple regression analysis to predict attribution styles from factor structure

\begin{tabular}{llcccccc}
\hline \multicolumn{1}{c}{ Dependent variable } & Independent variable & $\mathrm{B}$ & $\mathrm{SE}$ & $\mathrm{Beta}$ & $\mathrm{t}$ & $\mathrm{p}$ & Model's properties \\
\hline Hostility perception bias & Reflective self factor & -0.253 & 0.107 & -0.318 & -2.37 & 0.022 & $\mathrm{R}^{2}=0.101$, adj. $\mathrm{R}^{2}=0.083, \mathrm{~F}=5.61, \mathrm{p}=0.022$ \\
Composite Blame bias & Reflective self factor & -0.533 & 0.149 & -0.444 & -3.58 & 0.001 & $\mathrm{R}^{2}=0.262$, adj. $\mathrm{R}^{2}=0.233, \mathrm{~F}=8.90, \mathrm{p}<0.001$ \\
& Pre-reflective self factor & 0.342 & 0.118 & 0.361 & 2.909 & 0.005 & \\
\hline
\end{tabular}

Factor 3 as "neurocognition."

The respective correlations of the three factors with self-directedness, self-transcendence, and IQ were verified. Pearson's correlation analysis revealed that the factor scores of reflective self factor were significantly correlated with self-directedness, the scores of pre-reflective self factor were associated with self-transcendence, and the neurocognition factor was significantly correlated with IQ in the entire subject population (Table 4). Self-directedness additionally showed correlation with pre-reflective self factor.

\section{Association between AIHQ and the three factors in UHR individuals}

Preliminary Pearson's correlation analysis results are outlined in Supplementary Table 1 (in the online-only Data Supplement). In the UHR group, there was one significant correlation between hostility perception bias and reflective self factor. For composite blame bias, UHR individuals showed that reflective self factor and pre-reflective self factor were significantly correlated. Other than this, there were no significant correlations.

Results of multiple regression analysis to predict attribution styles from the factor structure are shown in Table 5. Regression analysis with hostility perception bias as the dependent variable revealed that reflective self factor was a significant determinant that explained $8.3 \%$ of the variance. In the regression model of composite blame bias, there were independent predictors including reflective self factor and pre-reflective self factor, which accounted for $23.3 \%$ of the variance.

\section{DISCUSSION}

The main findings of the current study were that 54 individuals at UHR for psychosis showed significantly biased attribu- 
tion styles of hostility perception and blaming and more importantly, the biased attribution styles were associated with two aspects of self. To our best knowledge, this is the first study to investigate the associations of multifaceted self-related psychological and neurocognitive performance variables with attribution bias in individuals for UHR for psychosis. The associations have been demonstrated in two steps. First, three factors were determined with exploratory factor analysis of the data on 13 multifaceted self-related psychological and neurocognitive variables of the entire sample population (UHR individuals and HCs). These three factors were designated as reflective self, pre-reflective self, and neurocognition, and all of these showed respective correlations with other variables such as self-directedness, self-transcendence, and IQ. Second, the evaluation of the relationship between biased attribution style and the three factors in UHR individuals revealed that perceived hostility bias was associated with the reflective self factor, and blame bias was independently associated with the pre-reflective and reflective factors.

The UHR group demonstrated greater hostility perception and blame biases, which is consistent with our previous study. ${ }^{5}$ The increased tendencies to perceive hostility and to blame others may be related to negative expectation. The expected malevolent wishes of others could possibly have originated from past experiences of more frequent negative emotion, which was partially deduced from home videos of preschizophrenic childhood period..$^{59}$

The three factor structure was revealed from the factor analysis of the data of multifaceted self-related psychological and neurocognitive performance variables. All the items which loaded on each factor i.e., resilience, self-perception, self-esteem, social anhedonia, and physical anhedonia on the reflective self factor; magical ideation, perceptual aberration, and basic symptoms on the pre-reflective self factor; all neurocognitive performance variables on the neurocognition factor, were grossly compatible with the properties of reflective self, pre-reflective self, and neurocognition. Despite the higher loading on pre-reflective self factor (Factor score $=0.75$ ), the basic symptom variable was also loaded to reflective self factor (Factor score $=-0.52$ ). This finding is compatible with that of the earlier studies indicating that the pre-reflective sense of self serves as an essential foundation for reflective representation of self. ${ }^{30}$ While perceptual aberration and magical ideation out of four components of schizotypy loaded on the pre-reflective self factor as expected, social anhedonia and physical anhedonia loaded on the reflective self factor. In a previous study, subjective experiences of basic symptoms measured using FCQ were not associated with anhedonia components but were associated with perceptual aberration and magical ideation of schizotypy. ${ }^{32}$ These results may be possibly explained by the characteristics of the questionnaires for social anhedonia and physical anhedonia. In the Chapman's social anhedonia and physical anhedonia scales, participants are provided with hypothetical scenarios and asked to think how they would feel in those situations. The hypothetical self-reports of anhedonia reflect the participants' selfrelated belief, ${ }^{60}$ and it seems to correspond to the intrinsic component of reflective selfhood.

The relationship between the biased attribution styles and the three factors showed the influence of various aspects of self on attribution style in the UHR individuals. Higher hostility perception bias in the UHR individuals was associated with a lower factor score of reflective self factor. A low loading on reflective self factor indicates low self-efficacy, negative self-perception, low self-esteem, and high anhedonia, which in turn may lead the person to perceive oneself as fragile. ${ }^{26}$ This finding is also comparable to previous findings ${ }^{13,17}$ of negative self-representation eliciting external personal attributions to prevent the feeling of discrepancies between the ideal and actual representations of self.

For increased composite blame bias in the UHR individuals, both pre-reflective and reflective self were associated. The associations may be explained by considering the components of composite blame bias i.e., intentionality, blame, and anger. The relationship between the blame bias and reflective self factor may be understood in the same manner as explained earlier that negative awareness of self induces the attribution of intentionality. The function of another independent predictor of composite blame bias, pre-reflective self factor, may be comprehended by its link to emotional distress and emotional reaction. ${ }^{26}$ Anomalous subjective experiences provoke anxiety due to a feeling of ontological vulnerability, which makes it difficult for the person to maintain boundaries around the self. ${ }^{61}$ In this state, any perceived attack by malevolent others may be regarded as an imminent catastrophic threat, and the distress could cause emotional reactions such as fear or anger. $^{26,61}$

The association of the attribution style with neurocognition, contrary to our hypothesis, was not found in this study. The possible explanation is that the domains of neurocognitive performance that were assessed in this study may actually not be associated with the attribution style. Otherwise, in accordance with the result that the neurocognitive deficit in UHR individuals was not substantial, the influence of neurocognition factor could not be found since the attribution questionnaire used in current study may not have been administered under the cognitive busy condition. Further studies on cognitively high loading attribution tasks or UHR individuals with severe neurocognitive deficits may contribute to the progress in our understanding. 
A less inclined aggressive response bias in UHR individuals was observed while the hostility perception and blame biases were significantly increased. The emotional reaction may not always evoke aggressive responses since others' response may be fearful, but may provoke the expression of aggression if the person possess high self-efficacy to beat others. ${ }^{26}$ The difference between our finding of decreased aggressive response bias in UHR individuals and the increased aggression bias of chronic schizophrenia patients 4 could be related to the above distinct character of anxiety-related processes and safety behaviors in each group.

Limitations of the present study should be considered when interpreting our results. One limitation is the cross-sectional nature of our data. To increase the understanding of the causal relationships of attribution style and the multifaceted selfrelated psychological variables and neurocognitive performance, long-term follow-up studies may be required. Multiple correlations could also be considered as limitations because they may increase false positive findings. Although we set the criterion for significance to Bonferroni-corrected $\mathrm{p}<0.05$, the preliminary findings of the correlations of biased attribution style and the three factors require cautious interpretation.

In conclusion, this study showed that biased attribution styles were exhibited in individuals at UHR for psychosis, and these were associated with multifaceted self-related psychological constructs in the UHR individuals. By using comprehensive variables related to attribution style, we could understand the overall picture of the psychological components of attribution in a 3-dimensional perspective. These findings indicate that various psychological interventions (e.g., cognitive-behavioral therapy, ${ }^{62}$ and social cognition training program $^{63}$ ) should include a session focusing on not only the reflective self but also pre-reflective self-related psychological constructs to deal with biased attribution styles, which would then reduce intrapersonal and interpersonal distress in individuals with UHR for psychosis.

\section{Supplementary Materials}

The online-only Data Supplement is available with this article at https://doi.org/10.30773/pi.2018.05.08.

\section{Acknowledgments}

This work was supported by the Basic Science Research Program through the National Research Foundation of Korea (NRF) funded by the Ministry of Science, ICT \& Future Planning, Republic of Korea (Grant number: 2017R1A2B3008214).

\section{REFERENCES}

1. Bentall RP, Corcoran R, Howard R, Blackwood N, Kinderman P. Persecutory delusions: a review and theoretical integration. Clin Psychol Rev 2001;21:1143-1192.

2. Kinderman P, Bentall RP. Causal attributions in paranoia and depres- sion: internal, personal, and situational attributions for negative events. J Abnorm Psychol 1997;106:341-345.

3. Garety PA, Freeman D. Cognitive approaches to delusions: a critical review of theories and evidence. Br J Clin Psychol 1999;38:113-154.

4. Combs DR, Penn DL, Michael CO, Basso MR, Wiedeman R, Siebenmorgan $\mathrm{M}$, et al. Perceptions of hostility by persons with and without persecutory delusions. Cogn Neuropsychiatry 2009;14:30-52.

5. An SK, Kang JI, Park JY, Kim KR, Lee SY, Lee E. Attribution bias in ultra-high risk for psychosis and first-episode schizophrenia. Schizophr Res 2010;118:54-61.

6. Astington JW, Harris PL, Olson DR. Developing Theories of Mind. Cambridge: Cambridge University Press; 1990.

7. Gallagher S. Neurocognitive models of schizophrenia: a neurophenomenological critique. Psychopathology 2004;37:8-19.

8. Robinson MD, Clore GL. Belief and feeling: evidence for an accessibility model of emotional self-report. Psychol Bull 2002;128:934-960.

9. Bentall RP, Kinderman P, Moutoussis M. The Role of Self-Esteem in Paranoid Delusions: The Psychology, Neurophysiology, and Development of Persecutory Beliefs. In: Freeman D, Bentall R, Garety P, Editors. Persecutory Delusions. Oxford: Oxford University Press, 2008, p.143173.

10. Freeman D, Garety PA, Fowler D, Kuipers E, Bebbington PE, Dunn G. Why do people with delusions fail to choose more realistic explanations for their experiences? An empirical investigation. J Consult Clin Psychol 2004;72:671-680.

11. Frith CD. The Cognitive Neuropsychology of Schizophrenia. London: Taylor \& Francis; 2014.

12. Green MF. Schizophrenia from a Neurocognitive Perspective: Probing the Impenetrable Darkness. Needham Heights, MA, US: Allyn \& Bacon; 1998.

13. Bentall RP. Cognitive Biases and Abnormal Beliefs: Towards a Model of Persecutory Delusions. In: David AS, Cutting J, Editors. The Neuropsychology of Schizophrenia. Hove, UK: Lawrence Erlbaum Associates, 1994, p.337-360.

14. Peterson C, Semmel A, Von Baeyer C, Abramson LY, Metalsky GI, Seligman ME. The attributional style questionnaire. Cog Ther Res 1982;6: 287-299.

15. Haugen R, Lund T. Attributional style and its relation to other personality dispositions. Br J Educ Psychol 1998;68:537-549.

16. McKay R, Langdon R, Coltheart M. The defensive function of persecutory delusions: an investigation using the Implicit Association Test. Cogn Neuropsychiatry 2007;12:1-24.

17. Moritz S, Werner R, von Collani Gv. The inferiority complex in paranoia readdressed: a study with the Implicit Association Test. Cogn Neuropsychiatry 2006;11:402-415.

18. Nelson B, Whitford T, Lavoie S, Sass L. What are the neurocognitive correlates of basic self-disturbance in schizophrenia?: integrating phenomenology and neurocognition. Part 1 (Source monitoring deficits). Schizophr Res 2014;152:12-19.

19. Raballo A, Parnas J. The silent side of the spectrum: schizotypy and the schizotaxic self. Schizophr Bull 2011;37:1017-1026.

20. Schultze-Lutter F. Subjective Symptoms of schizophrenia in research and the clinic: the basic symptom concept. Schizophr Bull 2009;35:5-8.

21. Lenzenweger MF, Korfine L. Tracking the Taxon: On the Latent Structure and Base Rate of Schizotypy. In: Raine A, Lencz T, Mednick SA, Editors. Schizotypal Personality. Cambridge: Cambridge University Press, 1995, p.135-167.

22. Moore JW, Pope A. The intentionality bias and schizotypy. Q J Exp Psychol (Hove) 2014;67:2218-2224.

23. Villatte M, Monestès JL, McHugh L, i Baque EF, Loas G. Adopting the perspective of another in belief attribution: contribution of relational frame theory to the understanding of impairments in schizophrenia. J Behav Ther Exp Psychiatry 2010;41:125-134.

24. Lee SY, Bang M, Kim KR, Lee MK, Park JY, Song YY, et al. Impaired facial emotion recognition in individuals at ultra-high risk for psychosis 
and with first-episode schizophrenia, and their associations with neurocognitive deficits and self-reported schizotypy. Schizophr Res 2015; 165:60-65.

25. Mass R, Weigel S, Schneider S, Klepsch R. Schizophrenia-specific basic symptoms. A successful replication. Psychopathology 1998;31:113-119.

26. Salvatore G, Lysaker PH, Popolo R, Procacci M, Carcione, A Dimaggio G. Vulnerable self, poor understanding of others' minds, threat anticipation and cognitive biases as triggers for delusional experience in schizophrenia: a theoretical model. Clin Psychol Psychother 2012;19: 247-259.

27. Esslen M, Metzler S, Pascual-Marqui, R Jancke L. Pre-reflective and reflective self-reference: a spatiotemporal EEG analysis. Neuroimage 2008; 42:437-449.

28. Nelson B, Fornito A, Harrison BJ, Yucel M, Sass LA, Yung AR, et al. A disturbed sense of self in the psychosis prodrome: linking phenomenology and neurobiology. Neurosci Biobehav Rev 2009;33:807-817.

29. Gallagher I. Philosophical conceptions of the self: implications for cognitive science. Trends Cogn Sci 2000;4:14-21.

30. Henriksen MG, Nordgaard J. Self-Disorders in Schizophrenia. In Stanghellini G, Aragona M, Editors. An Experiential Approach to Psychopathology. What Is It Like to Suffer from Mental Disorders. New York; Springer, 2016, p.265-280.

31. Bechdolf A, Schultze-Lutter F, Klosterkötter J. Self-experienced vulnerability, prodromal symptoms and coping strategies preceding schizophrenic and depressive relapses. Eur Psychiatry 2002;17:384-393.

32. Yon V, Loas G, Monestès JL. Relationships between schizotypy and subjective experiences in a sample of 399 university students. Compr Psychiatry 2009;50:142-150.

33. Fusar-Poli P, Deste G, Smieskova R, Barlati S, Yung AR, Howes O, et al. Cognitive functioning in prodromal psychosis: a meta-analysis. Arch Gen Psychiatry 2012;69:562-571.

34. Yung AR, McGorry PD. The prodromal phase of first-episode psychosis: past and current conceptualizations. Schizophr Bull 1996;22:353370.

35. Nelson B, Raballo A. Basic self-disturbance in the schizophrenia spectrum: taking stock and moving forward. Psychopathology 2015;48:301309.

36. DeVylder JE, Ben-David S, Kimhy D, Corcoran CM. Attributional style among youth at clinical risk for psychosis. Early Interv Psychiatry 2013;7:84-88.

37. Thompson A, Papas A, Bartholomeusz C, Nelson B, Yung A. Externalized attributional bias in the Ultra High Risk (UHR) for psychosis population. Psychiatry Res 2013;206:200-205.

38. First MB, Spitzer RL, Miriam G, Williams JBW. Structured Clinical Interview for DSM-IV Axis I Disorders: Non-Patients Edition (SCID-I/ PS), Version 2. New York: New York State Psychiatric Institute Biometric Research; 1996.

39. First MB, Spitzer RL, Miriam G, Williams JBW. Structured Clinical Interview for DSM-IV Axis I Disorders: Patient Edition (SCID-I/P). Version 2. New York: New York State Psychiatric Institute Biometric Research; 1996.

40. McGlashan T, Miller T, Woods S, Rosen J, Hoffman R, Davidson L. Structured Interview for Prodromal Syndromes (SIPS). Version 4.0. New Heaven: Yale University; 2003.

41. Connor KM, Davidson JR. Development of a new resilience scale: The Connor-Davidson Resilience Scale (CD-RISC). Depress Anxiety 2003; 18:76-82.

42. Kim JH, Lee CH. Relations of perceived stress, cognitive set, and coping behaviors to depression: a focus on freshmen's stress experiences. Korean J Counsel Psychother 1988;1:25-45.

43. Roserberg M. Society and the Adolescent Self-Image, Revised Edition. Middletown, CT, USA: Wesleyan University Press; 1989.

44. Chapman L, Chapman J. Revised Physical Anhedonia Scale. Madison: University of Wisconsin; 1978.

45. Eckblad M, Chapman L, Chapman J, Mishlove M. The Revised Social Anhedonia Scale. Madison: University of Wisconsin; 1982.

46. Eckblad M, Chapman LJ. Magical ideation as an indicator of schizotypy. J Consult Clin Psychol 1983;51:215-225.

47. Chapman LJ, Chapman JP, Raulin ML. Body-image aberration in schizophrenia. J Abnorm Psychol 1978;87:399-407.

48. Süllwold L. Frankfurter Beschwerde-Fragebogen (FBF). In: Süllwold L, Huber G, Editors. Schizophrene Basisstörungen. Berlin, Germany: Springer; 1986.

49. Bang M, Kim KR, Song YY, Baek S, Lee E, An SK. Neurocognitive impairments in individuals at ultra-high risk for psychosis: who will really convert? Aust N Z J Psychiatry 2015;49:462-470.

50. Kim KR, Park JY, Song DH, Koo HK, An SK. Neurocognitive performance in subjects at ultrahigh risk for schizophrenia: a comparison with first-episode schizophrenia. Compr Psychiatry 2011;52:33-40.

51. Chang H, Lee SK, Kim KR, Lee SY, Park JY, Kim EJ, et al. Development of Korean version of the ambiguous intentions Hostility Questionnaire (K-AIHQ). J Korean Neuropsychiatr Assoc 2009;48:29-35.

52. Combs DR, Penn DL, Wicher M, Waldheter E. The Ambiguous Intentions Hostility Questionnaire (AIHQ): a new measure for evaluating hostile social-cognitive biases in paranoia. Cogn Neuropsychiatry 2007; 12:128-143.

53. Cloninger CR, Przybeck TR, Svrakic DM. The Temperament and Character Inventory (TCI): A Guide to its Development and Use. St. Louis, Mo: Center for Psychobiology of Personality, Washington University; 1994.

54. Goth K, Cloninger CR, Schmeck K. Das Temperament und Charakter Inventar Kurzversion fur Erwachsene-TCI R Kurz. Frankfurt: J. W. Goethe University, Dept. of Psychology; 2003.

55. Min B, Oh H, Lee J. Temperament and Character Inventory-Family Manual. Seoul: Maumsarang Co.; 2007.

56. Boeker H, Kleiser M, Lehman D, Jaenke L, Bogerts B, Northoff G. Executive dysfunction, self, and ego pathology in schizophrenia: an exploratory study of neuropsychology and personality. Compr Psychiatry 2006;47:7-19.

57. Kim J, Lee Y. Validity of short forms of the Korean-Wechsler adult intelligence scale. Korean J Clin Psychol 1995;14:111-116.

58. Kay SR, Fiszbein A, Opfer LA. The positive and negative syndrome scale (PANSS) for schizophrenia. Schizophr Bull 1987;13:261-276.

59. Walker EF, Grimes KE, Davis DM, Smith AJ. Childhood precursors of schizophrenia: facial expressions of emotion. Am J Psychiatry 1993;150: 1654-1660.

60. Strauss GP, Gold JM. A new perspective on anhedonia in schizophrenia. Am J Psychiatry 2012;169:364-373.

61. Lysaker PH, Lysaker JT. Schizophrenia and the Fate of Self. Oxford: Oxford University Press; 2008.

62. Morrison AP, French P, Walford L, Lewis SW, Kilcommons A, Green J, et al. Cognitive therapy for the prevention of psychosis in people at ultra-high risk. Br J Psychiatry 2004;185:291-297.

63. Penn DL, Roberts DL, Combs D, Sterne A. Best practices: the development of the social cognition and interaction training program for schizophrenia spectrum disorders. Psychiatr Serv 2007;58:449-451. 